\title{
Adherence to iron and folic acid supplementation and associated factors among antenatal care attendants in Northwest Ethiopia
}

\author{
Berhanu Abebaw ${ }^{1}$, Yadeta Dessie ${ }^{2}$, Negga Baraki ${ }^{3}$, and Abdu Oumer ${ }^{4}$ Mehari Gebru ${ }^{5}$ \\ ${ }^{1}$ Department of Nutrition and Dietetics, School of Public Health, Bahir Dar University, Ethiopia \\ ${ }^{2,3}$ Department of Public Health, College of Health and Medical Sciences, Haramaya University, Ethiopia \\ ${ }^{4,5}$ Department of Public Health, College of Health Science and Medicine, Wolkite University, Ethiopia
}

\begin{tabular}{l}
\hline \hline Article Info \\
\hline Article history: \\
Received Nov 15, 2019 \\
Revised Jan 11, 2020 \\
Accepted Feb 15, 2020 \\
\hline
\end{tabular}

\section{Keywords:}

Adherence

Debre Markos

Iron-folic Acid

Pregnant women

\begin{abstract}
Iron and folic acid supplementation is the key approach for anemia prevention and control during pregnancy. In Ethiopia only <1\% of pregnant mothers ingest the ideal number of tablets. Although, adherence is the most important challenge, literature is dearth and the predictors are undoubtedly recognized. Institution based quantitative cross sectional study design triangulated with qualitative methods was employed among 395 systematically selected pregnant mothers attending antenatal care in Debre Markos town, Ethiopia. Data were collected using interviewer administered structured questionnaire. Data were entered into Epi data and exported to SPSS software. Bivariate and multivariable Logistic regression with the $95 \%$ confidence interval was computed. P-value $<0.05$ was declared as statistically significant. Eight in-depth interviews were conducted. The data were entered and analyzed using open code software. Adherence rate was $55.5 \%$ (95\% CI, 50.5\%-60.4\%). Pregnant mothers who had; history of anemia during current pregnancy [AOR:7.9, 95\%CI (4.44-14.01)], primary education (AOR:4.0, 95\% CI (1.88-8.54)], secondary education and above (AOR:3.6, 95\%CI (1.20-6.94)], good knowledge of iron and folic acid supplementation [AOR:2.1, 95\% CI (1.24-3.56)], and early registration for antenatal care (AOR:1.8, 95\% CI (1.06-3.11)] were predictors of iron and folic acid supplementation adherence. The rate of adherence was low. Getting medical advice and fear of illness if missed were the primary reasons that enforce mothers to take the tablets. Hence, improving mothers' knowledge regarding overall aspects of the tablet through better advice, community teaching and the mass media at large, would improve adherence.
\end{abstract}

This is an open access article under the CC BY-SA license.

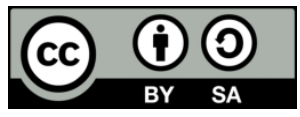

\section{Corresponding Author:}

Berhanu Abebaw,

Department of Nutrition and Dietetics,

School of Public Health,

Bahir Dar University, Ethiopia.

Email: birhanua20@gmail.com

\section{INTRODUCTION}

Anemia refers to a condition in which the existing red blood cells are inadequate to satisfy the body's physiologic demands. It affects around one fourth of the world's population. Anemia affects more than two fifth of pregnant mothers worldwide [1]. Approximately 20\% of pregnant women in developed countries and more than 50\% in developing countries are affected by iron deficiency [2]. According to 
the 2011 Ethiopian Demographic and health surveys, more than one fifth of pregnant women were affected by anemia [3]. Anemia in pregnancy was found to be a leading cause of morbidity and mortality, both for the mother and for the developing baby [4].

Iron is a trace element that is necessary for hemoglobin synthesis. Highly cost effective iron intervention is delivering iron together with folic acid in tablets (IFAS) to pregnant mothers. But the effectiveness of such interventions depends on the adherence to the tablets [5]. Foliate deficiency is associated with anemia and other unintended outcomes in pregnancy. Folic acid supplementation is currently among the cost effective ways recommended to control those outcomes [6].

According to WHO and Ethiopian guidelines, all pregnant mothers should take 30-60 mg iron plus $400 \mu \mathrm{g}$ folic acid, one supplement daily starting as early as possible and taken the whole pregnancy period [7,8]. Despite its implementation, many countries are not effective in anemia control and prevention due to poor compliance of mothers to IFAS [5, 6]. Adherence to a medicine refers to the degree to which patients take medicines/tablets as prescribed by their care givers. Adherence rates are commonly described as the proportion of the prescribed doses of the medicine/tablet truly ingested by the patient over a definite period.

One in every three women had anemia and deficiency of folic acid while about half had iron deficiency, indicating that such conditions are found to be the most important micronutrient deficiencies in Ethiopia [9]. Numerous countries (developing) are now employing iron/folic acid supplementation programs, but only a few countries had substantial progress in anemia prevention and control. Various studies from different parts of the world (Asia, Latin America and African countries) have shown low adherence of women taking daily IFAS and this takes the lions share for IFAS programs ineffectiveness. There are different factors that can affect the adherence status, which are not studied extensively [5, 10, 11].

In Ethiopia, IFAS is the key approach for anemia control and prevention. However, adherence rate persists to be very low. A study conducted in Ethiopia showed that only about one in sex pregnant mothers took the IFA supplements for three months or more [8]. In another study in Ethiopia, only less than $1 \%$ ingests the ideal number of tablets (minimum of 180 IFA tablets) [12]. More than eight out of ten women, more than one in six women and less than one in hundred women; didn't took iron tablets at all, took the tablets for less than 60 days and took the tablets for three months or more during their last pregnancy respectively [3]. Although, adherence is the most important challenge in IFAS programs, literature is dearth and the predictors are not persistent and undoubtedly recognized. Therefore, this study was aimed to assess the adherence status (IFAS) and its predictors among pregnant mothers attending ANC in Debre Markos town administration, Ethiopia.

\section{RESEARCH METHOD}

\subsection{Study setting}

The study was conducted in Debre Markos town Administration, Northwest Ethiopia from March 03-23/2016. The town is located about 300 kilometres Northwest from Addis Ababa, Ethiopia. The town has a total of eight kebeles, and bordered by Gozamen District. The estimated total population of the town is 68,960 of which male populations constitute $33,291(48.2 \%)$ and female accounts for $35,669(51.8 \%)$. Regarding health services, the town administration has one referral hospital, three health centres, eight health posts, four small and six medium private clinics, two private drug vendors and four private drug stores. Institution based quantitative cross sectional study design triangulated with qualitative methods was employed among 395 systematically selected pregnant mothers from the selected health facilities. All pregnant women who took IFAS at least for a month and visited the ANC clinic during the study period were the source populations.

\subsection{Sample size determination}

The final sample size was determined after calculating and comparing the sample size for the two specific objectives; accordingly the maximum sample size was obtained by using single population proportion estimation formula (Normal deviant at the portion of 95\% confidence interval two tailed test $=1.96$; margin of error acceptable is taken as 5\%) and taking the rate of adherence to IFAS among pregnant mothers as $37.2 \%$ from a study done in a similar set up [13]. After adding $10 \%$ non-response rate, finally the total sample size was 395. In the qualitative study six pregnant mothers and two key informants (heads of ANC clinics) participated in in-depth interviews.

In Debre Markos Town Administration, there are three public health centers and one referral hospital. Namely, Hedasie health center, Debre Markos health center, Wuseta health center and Debre Markos referral hospital. In all health institutions there were pregnant women attending ANC clinic. All of those institutions were involved in the study. By using the flow of pregnant mothers 
visiting ANC clinic in the previous months as a baseline there were a total of 792 pregnant women in those public health facilities, who fulfill the eligibility criteria during the study period. Considering the total number of pregnant mothers $(\mathrm{N}=792)$, and the sample size $(\mathrm{n}=395)$, an interval of $\mathrm{k}=\mathrm{N} / \mathrm{n}=792 / 395=2$, was used to select the study subjects. The sample size was proportionally allocated to each health facility and the participants were selected using systematic random sampling technique.

\subsection{Data collection tools and data collectors}

Data were collected using translated structured questionnaire adapted from Dinga et al., and further developed by using different peer reviewed published literatures. The questionnaire addressed the participant's socio-demographic factors; Obstetric and health related characteristics, Health service related factors; Client related factors (knowledge) and others. The questionnaire was modified further after a pre-test was conducted. Separate eight in-depth interview guide was prepared in English language for both pregnant women and key informants from previous literatures. Then it was translated into local Amharic language. The in-depth interview guide included general information about the respondents and other open ended questions that focus on reasons for adherence/non adherence to IFAS. Four grade ten completed girls were recruited to participate in data collection. The data collectors were selected from Debre Markos town administration, who were fluent in local language. One Public Health officer was recruited for supervision. In order to collect the qualitative data, the principal investigator conducted in-depth interviews on six pregnant women and two key informants. Open ended questions with multiple probing were used to get adequate information. The interviews were recorded via tape recorder.

\subsubsection{Variables of the study}

The dependent variable was IFAS adherence. The independent variables were socio demographic and economic factors, obstetric characteristics, knowledge related factors and others.

\subsubsection{Data quality control}

The data collectors had taken two days training. The questionnaire was translated into the local language of the respondents (Amharic language) and used for the data collection. Pre test was done and necessary amendments were made in the tool. During the actual data collection, close supervision was made and accordingly, data were checked for its consistency and completeness. Double entry was made to cross check the data for completeness before analysis. For the qualitative data, the already conducted interviews were analysed before the next interview for avoidance of unnecessary data collection and possible amendment of the tool.

\subsubsection{Data processing and analysis}

After checking the collected data visually, data were coded, entered and cleaned using Epi-Data version 3.3.1 software. The data entered was exported to SPSS version 23 software for analysis. Frequency and percentages were calculated to describe the different characteristics of the study subjects. Bivariate logistic regression was performed for each independent variable with outcome variable. A variable whose bivariate test had a p-value $<0.2$ was a candidate for multivariable model together with all other variables. The Hosmer and Lemeshow's goodness-of-fit test model coefficient was considered to assess whether the necessary assumptions for the application of multiple logistic regression were fulfilled. Multivariable logistic regression was performed to identify the predictors of IFAS adherence. Multicollinearity was checked and all non collinear variables were taken to the model. Crude and adjusted odds ratios were calculated; all tests were two-sided and $\mathrm{P}$ value $<0.05$ was considered statistically significant. For the qualitative data analysis Open code software was used. First the tape recorded audio data was transcribed to paper in Amharic language and further translated to English. Then it was entered to computer in word document. Thereafter it was exported to open code software for descriptive content analysis.

\subsection{Ethical considerations}

Officially written approval letter from Haramaya University Institutional Health Research Ethics Review Committee (IHRERC) was obtained prior to the data collection. Before informed consent obtained, the clear description of the study title, procedure and duration, possible risks and benefits of the study were explained for the study subjects. and the responses of interviewee`s were kept confidential. 


\section{RESULTS AND DISCUSSION}

About 384 pregnant mothers were participated yielding response rate of 97.2\%. About $233(60.7 \%)$ study participants were age $\geq 25$ years. Three hundred twenty five $(84.6 \%)$ of study participants resided in urban. The vast majority, 351(91.4\%) were Orthodox Christian followers and 365(95.1\%) of the participants were married. About $186(48.4 \%)$ of the study participants were house wife followed by government employee $78(20.3 \%)$, and $243(63.3 \%)$ of respondents had family size of at most three. Frequency of Socio-economic and demographic characteristics of respondents in Debre Markos town, Northwest Ethiopia, March 2016, (N=384) shown in Table 1.

Table 1. Frequency of Socio-economic and demographic characteristics of respondents

\begin{tabular}{|c|c|c|c|}
\hline Variable & Category & Frequency & Percent \\
\hline \multirow{2}{*}{ Age } & $<25$ years & 151 & 39.3 \\
\hline & $\geq 25$ years & 233 & 60.7 \\
\hline \multirow{2}{*}{ Religion } & Orthodox & 351 & 91.4 \\
\hline & Others* & 33 & 8.6 \\
\hline \multirow{2}{*}{ Residence } & Urban & 325 & 84.6 \\
\hline & Rural & 59 & 15.4 \\
\hline \multirow{4}{*}{ Educational status } & Cannot read and write & 131 & 34.1 \\
\hline & Can read and write & 67 & 16.4 \\
\hline & Primary education & 64 & 17.7 \\
\hline & Secondary and above & 122 & 31.8 \\
\hline \multirow{5}{*}{ Occupational status } & Government employee & 78 & 20.3 \\
\hline & Daily laborer & 30 & 7.8 \\
\hline & Merchant & 70 & 18.2 \\
\hline & House wife & 186 & 48.4 \\
\hline & Others** & 20 & 5.2 \\
\hline \multirow{2}{*}{ Marital Status } & Married & 365 & 95.1 \\
\hline & Others $* * *$ & 15 & 4.9 \\
\hline \multirow{4}{*}{ Husband Educational status } & Cannot read and write & 103 & 27.8 \\
\hline & Can read and write & 58 & 15.7 \\
\hline & Primary education & 42 & 11.4 \\
\hline & Secondary and above & 167 & 45.1 \\
\hline \multirow{3}{*}{ Family size } & $1-3$ & 243 & 63.3 \\
\hline & $4-6$ & 121 & 31.5 \\
\hline & $>6$ & 20 & 5.2 \\
\hline \multirow{3}{*}{ Family income } & $<500$ birr & 59 & 15.4 \\
\hline & 500-1000birr & 98 & 25.5 \\
\hline & $>1000$ birr & 227 & 59.1 \\
\hline
\end{tabular}

The mean ( \pm SD) gestational age of the respondents' was 27.6 \pm 7.6 weeks. About 225(58.6\%) of the respondents were primigravidas and only 143(37.2\%) were Multiparous. More than half 207(53.9\%) of the participants were in their third trimester. About 260(67.7\%) of the respondents had ANC follow up within their 16weeks of gestation. More than ninety percent (354) of the respondents had at most four ANC visits. About 223(58.1\%) of the participants spent more than half an hour (by foot) to reach the health facility and $365(94.5 \%)$ of the participants got medical advice about IFAS. More than one fifth $(21.6 \%)$ and more than one third $(37.5 \%)$ of the participants had history of previous anemia and history of anemia during current pregnancy respectively Table 2.

Table 2. Frequency of Obstetric and health facility related characteristics of respondents

\begin{tabular}{cccc}
\hline Variable & Category & Frequency & Percent \\
\hline \multirow{2}{*}{ Gravidity } & Primigravida & 159 & 41.4 \\
& Multigravida & 225 & 58.6 \\
& Nulliparous & 168 & 43.8 \\
Parity & Primiparous & 73 & 19.0 \\
& Multiparous & 143 & 37.2 \\
& First & 39 & 10.2 \\
Trimester & Second & 138 & 35.9 \\
& Third & 207 & 53.9 \\
Time of & <16 weeks & 260 & 67.7 \\
registration & (Early) & &
\end{tabular}

\begin{tabular}{cccc}
\hline Variable & Category & Frequency & Percent \\
\hline Number of Visits & $\leq 4$ & 354 & 92.2 \\
& $>4$ & 30 & 7.8 \\
Distance from health & $\leq 30$ & 161 & 41.9 \\
facility & minutes & & \\
& $>30$ & 223 & 58.1 \\
Get Medical advice about & minutes & 363 & 94.5 \\
IFAS & Yes & 21 & 5.5 \\
History of previous anemia & No & 83 & 21.6 \\
History of anemia during & No & 301 & 78.4 \\
current pregnancy & Yes & 144 & 37.5 \\
& No & 240 & 62.5 \\
\hline
\end{tabular}


Adherence assessment was made based on participants self-report of taking average number of IFA tablets (per week) during the previous one month preceding the survey. Two hundred thirteen [55.5\% (95\%CI, 50.5\%-60.4\%)] of participants were adhered to IFAS (took $\geq 4$ tablets per week) and $171[44.5 \%(95 \% \mathrm{CI}, 39.6-49.5)]$ were non-adhered to IFAS (took < 4 tablets per week). The leading reason for adherence was getting medical advice $(352,94.1 \%)$ followed by fear of illness if missed $(177,47.3 \%)$ and getting family support $(68,18.2 \%)$. From those who got medical advice, $(345,95.3 \%)$, $(324,89.5 \%)$ and $(54,14.9 \%)$ were advised about the importance of the supplement, how it is taken and side effect of the supplement respectively. Findings from the qualitative part of the study also shown that most pregnant mothers' leading motive of consuming the tablets was getting medical advice from health care providers.

..."I took the tablets because the health care providers (both at health facility and home) told me it prevents you from anemia and your fetus from diseases".

(Pregnant women, age 38)

Another qualitative finding showed that fear of illness (if tablets are missed) was the second major reason to taking the tablets.

..."I am forced to take the tablets because I fear the illness that would happen to me and to my fetus, if I missed the doses of tablets"

(Pregnant women, age 27)

Another reason for taking tablets raised by in-depth interview participants was getting family support.

...'I never missed the tablet, because my husband reminds me to take it".

(Pregnant women, age 38)

The primary reason for mothers who didn't ingest the tables, was forgetfulness $(248,67.8 \%)$ followed by fear of side effect $(190,51.9 \%)$. In addition, belief that taking tablets leads to too big baby $(133,36.3 \%)$ and taking a lot of tablets would injure the mother and infant $(38,10.4 \%)$ were reasons for missing the doses of tablets.

Findings from the qualitative component also revealed that forgetfulness was the major reason of missing tablets.

..."Since I forgot, I missed more than half of the tablets. Tablets are taken at night time, I spent all the day with work...when I returned back to home ... I will be so exhausted ... then I will forget them".

(Pregnant mother, age 32)

...'Pregnant mothers' first reason for missing the doses of tablets was forgetfulness, not side effect ... since we gave them counseling about side effects"

(ANC head, age 25)

Knowledge of anemia was calculated by adding 19 relevant knowledge questions (items on sign and symptom, cause, complication, prevention and others). A correct answer for each question was recorded as " 1 " and incorrect answer was recorded as " 0 ". Questions were add up and then converted to $100 \%$. Hence, the median was 47.4\%. About 167 (43.5\%) of the respondents had good knowledge of anemia (scored median and above) and $217(56.5 \%)$ of the respondents had poor knowledge of anemia (scored below the median). Similarly, knowledge of IFAS was computed by adding 7 relevant knowledge questions [14]. The median was $71.4 \%$. One hundred eighty one $(47.1 \%)$ of the respondents had good knowledge of IFAS and about 203 (52.9\%) of the respondents had poor knowledge of IFAS.

To determine the predictor variables, both bivariate and multivariable analysis were done. In the bivariate analysis, 14 variables: maternal educational status, partner educational status, occupational status, marital status, monthly family income, gravidity, trimester, time of ANC registration, number of ANC visit, history of previous anemia, history of anemia during current pregnancy, medical advice about IFAS, knowledge of anemia and knowledge of IFAS showed an association with IFAS at p value $<0.2$ and they were a candidate for the final model. Accordingly, maternal educational status, time of ANC registration, history of anemia during current pregnancy and knowledge of IFAS were found to be the predictor variables. The comparison of pregnant women those who adhered to IFAS and those who do not, revealed that; Pregnant mothers who had primary education were 4 times more likely to be adhered to IFAS compared to those who cannot read and write $(\mathrm{AOR}=4.0,95 \% \mathrm{CI}=1.88$ - 8.54). Pregnant women who were secondary education and above were 3.6 times more likely to be adhered to IFAS than those who cannot read and write $(\mathrm{AOR}=3.6,95 \% \mathrm{CI}=1.20-6.94)$. Pregnant mothers who had early registration $(\leq 16$ weeks $)$ for ANC were 1.8 times more likely to be adhered to IFAS than those who had late registration (>16weeks) (AOR=1.8, 95\% $\mathrm{CI}=1.06-3.11)$. Pregnant mothers who had good knowledge of IFAS were 2.1 times more likely to be adhered to IFAS than those who had poor knowledge of IFAS (AOR= 2.1, 95\% CI= 1.24-3.56). Pregnant mothers who had history of anemia during current pregnancy were 7.9 times more likely to be adhered to IFAS than those who hadn't $(\mathrm{AOR}=7.9,95 \% \mathrm{CI}=4.44-14.01)$ Table 3. 
Table 3. Multivariable logistic regression that shows predictors of IFAS adherence among pregnant mothers attending ANC clinics

\begin{tabular}{|c|c|c|c|c|}
\hline \multirow[t]{2}{*}{ Variables } & \multicolumn{4}{|c|}{ Adherence status } \\
\hline & $\begin{array}{r}\text { Adhered } \\
\text { № }(\%) \\
\end{array}$ & $\begin{array}{c}\text { Non adhered } \\
\text { № }(\%)\end{array}$ & $\mathrm{COR}(95 \% \mathrm{CI})$ & $\operatorname{AOR}(95 \% \mathrm{CI})$ \\
\hline \multicolumn{5}{|l|}{ Educational status } \\
\hline Cannot read and write & $44(20.7)$ & $87(50.9$ & & 1 \\
\hline Can read and write & $42(19.7)$ & $25(14.6)$ & $3.3(1.80,6.14)$ & $2.0(0.97,4.13)$ \\
\hline Primary education & $43(20.2)$ & $21(12.3)$ & $4.0(2.15,7.64)$ & $4.0(1.88,8.54)^{*}$ \\
\hline Secondary and above & $84(39.4)$ & $38(22.2)$ & $4.4(2.58,7.14)$ & $3.6(1.20,6.94)^{*}$ \\
\hline \multicolumn{5}{|l|}{ Monthly family income } \\
\hline$<500$ & 21(9.9) & $38(22.2)$ & 1 & \\
\hline $500-1000$ & $44(20.7)$ & $54(31.6)$ & $1.5(0.76,2.87)$ & $2.0(0.87,4.77)$ \\
\hline$>1000$ & $148(69.5)$ & $79(46.2)$ & $3.4(1.86,6.17)$ & $2.0(0.90,4.55)$ \\
\hline \multicolumn{5}{|l|}{ Gravidity } \\
\hline Primigravida & $82(38.5)$ & $77(45.0)$ & 1 & 1 \\
\hline Multigravida & $131(61.5)$ & $94(55.0)$ & $1.3(0.87,1.97)$ & $1.3(0.79,2.27)$ \\
\hline \multicolumn{5}{|l|}{ Trimester } \\
\hline First & $17(8.0)$ & $22(12.9)$ & 1 & 1 \\
\hline Second & $80(37.6)$ & $58(33.9)$ & $1.8(0.87,3.66)$ & $2.2(0.90,5.45)$ \\
\hline Third & $116(54.5)$ & $91(53.2)$ & $1.7(0.83,3.29)$ & $2.5(0.98,6.21)$ \\
\hline \multicolumn{5}{|l|}{ Time of registration } \\
\hline $\operatorname{Early}(\leq 16$ weeks $)$ & $170(79.8)$ & $90(52.6)$ & $3.6(2.27,5.58)$ & $1.8(1.06,3.11)^{* *}$ \\
\hline Late(>16weeks) & $43(20.2)$ & $81(47.4)$ & 1 & 1 \\
\hline \multicolumn{5}{|c|}{ History of anemia current pregnancy } \\
\hline Yes & $117(54.9)$ & $27(15.8)$ & $6.5(3.98,10.6)$ & $7.9(4.44,14.01)^{*}$ \\
\hline No & $96(45.1)$ & $144(84.2)$ & 1 & 1 \\
\hline \multicolumn{5}{|l|}{ Knowledge of Anemia } \\
\hline Poor & $102(47.9)$ & $115(67.3)$ & 1 & 1 \\
\hline Good & $111(52.1)$ & $56(32.7)$ & $2.2(1.47,3.39)$ & $1.4(0.80,2.48)$ \\
\hline \multicolumn{5}{|l|}{ Knowledge of IFAS } \\
\hline Poor & $98(46.0)$ & $105(61.4)$ & 1 & 1 \\
\hline Good & $115(54.0)$ & $66(38.6)$ & $1.9(1.24,2.81)$ & $2.1(1.24,3.56)^{* *}$ \\
\hline
\end{tabular}

Iron deficiency anemia greatly affects pregnant mothers. To prevent and mitigate such deficiencies, IFA supplementation is the realistic and practicable way. However adherence to IFAS is below the standard in most African countries including Ethiopia. The result revealed that $55.5 \%$ of pregnant women were adhered to Iron and folic acid supplementation (IFAS) which is consistent with the study done in Northwest Ethiopia (55.3\%) [14], West dembecha district, Ethiopia (52.9\%) [15] and in Indonesia (53.7\%) [16] However; it is higher than the study done in Mecha district, Northwest Ethiopia (20.4\%) [6], Tigray, Ethiopia (37.2\%) [13], Armachiho, Northwest Ethiopia (28.7\%) [17], Hawassa, South Ethiopia (38.3\%) [18], Adwa, Tigray Ethiopia (40.9\%) [19], Kenya (24.5\%) [20], Kiambu County, Kenya (32.7\%) [21]. The possible reason may be improved knowledge of mothers about anemia and IFAS, majority of the respondents being urban residents and the time gap between studies. The other possible rationale as told by qualitative study participants was; overestimation of taking tablets, since they did not exactly remind how much of the tablets were taken and how much were missed. The key informants also told that pregnant women favour overestimation of taking tablets because the health care providers ask them why if they miss the tablets, so in order not to be criticized by the providers', women tend to overestimate.

The study showed that women educational status was one of the predictor variables for IFAS adherence. Pregnant women who were having primary education were 4 times more likely to be adhered to IFAS compared to those who cannot read and write. The possible reason as told by the key informants was those who had primary education were eager to accept the counseling about IFAS. The finding is similar with other studies done in Northwest Ethiopia [6] Pakistan [22], Camboidia [23], and Indonesia [24]. However, this finding is inconsistent with other studies done in Kenya [20] and Sudan [25]. The variation may be due to geographical, and socio cultural (societal belief about IFAS) difference. On the other hand, pregnant mothers who were secondary education and above were 3.6 times more likely to be adhered to IFAS compared to those who were cannot read and write. This indicates that those women who had primary education were more likely to be adhered to IFAS than those who were secondary and above. The possible rationale as told by the qualitative study participants (key informants) were those who were secondary and above were prone to forgetfulness since most spent their time outside home with work: they did not want to suffer from side effects: think that they can get it (iron) just from diet and were reluctant to accept the counseling given by the health care provides, compared to those who had primary education. 
In this study history of anemia during current pregnancy had shown a significant association with IFAS adherence. Women who had history of anemia during current pregnancy were 7.9 times more likely to be adhered to IFAS than those who had not. The qualitative finding also revealed that, women were strict in taking the tablets if they had anemia because they need to be free from the illness due to fear of anemia complications both on them and their infant. The finding is consistent with other studies conducted in Mecha district, Northwest Ethiopia [6], Tigray, Ethiopia [13], Kenya [20] Tanzania [26], Debre tabor, Ethiopia [27].

Adherence rate was better seen in those mothers who were early registered for ANC services than those who were late registered. Pregnant mothers who were early registered were 1.8 times more likely to be adhered to IFAS than those who were late registered. The reason may be those who had early registration would had better attention for their prenatal period and had more ANC follow ups which will have effect for getting counseling and ultimatly improved knowledge about anemia and IFAS. The qualitative finding also revealed that, the early pregnant women visit the ANC clinic, the more they get adequate counsiling, and the better to adhere to the supplementation. The result is supported by the studies in India [11] and Ethiopia [12, 13]

Knowledge of IFAS was also another predictor; those women who had good knowledge IFAS were 2.1 times more likely to be adhered compared to those who had poor knowledge of IFAS. The likely justification may be, those who had good knowledge were having better opportunity about tablets benefit, side effects, when it has to be taken, and complication if missed. Findings from the qualitative component also showed that, women were encouraged to ingest the tablets if they have good knowledge about the tablets especially the benefits and side effects of the tablets and knowledge about IFAS can be increased by giving adequate counseling during medical advice and through health extension workers during home visit and community meetings. The finding is supported by studies done in Ethiopia [15, 19, 28].

There were different reasons for adherence and non-adherence to IFAS. Getting medical advice, and fear of illness if missed were the major reasons for mothers to be adhered to IFAS. This could be due to increased knowledge of both anemia (including pregnancy complications) and iron and folic acid supplementation that resulted from proper counseling through medical advice from health care providers. Qualitative study participants also told that they were willing to take the tablets if the health care providers were able to give medical advice about the tablets appropriately. Key informants also told that, even though medical advice is given, participants may not take the tablets because the quality of medical advice matters women to take the tablets. Therefore trainings should be given for the health care providers in order to deliver adequate counseling about IFAS. The finding is consistent with the study in Kenya [20]. The other reason of pregnant women for adherence was getting family support. The justification behind is that when pregnant women get family support, they will have an opportunity not to forget the tablets and great concern for adherence. Findings from the qualitative component also revealed that, family support especially husband support, play a great role for IFAS adherence because husbands consider the tablet as medication and think as it should be taken fully without missing, therefore they remind women to take the tablets. The finding is consistent with the study in Pakistan [22].

Forgetfulness was the primary reason of mothers for non-adherence to IFAS. A possible explanation for forgetfulness as a major reason is that the timing that the tablet is taken. Findings from the qualitative study revealed that since the tablet is taken at night, pregnant women were forced to forget the tablet since they the day time is loaded with various tasks and at night time they become tired. Lack of concern for adherence might also lead pregnant women to forget the tablets. The finding is consistent with other studies done in Ethiopia [12], Misha district, and South Ethiopia [29].

Following forgetfulness, fear of side effect was the prominent reason for non-adherence. Findings from the qualitative part also revealed that (as told by the key informants), women were prone to forget the tablets because they did not give emphasis for the tablets that resulted from reluctance of accepting advices, considering they can get the tablets benefit from diet and being busy with work. Fear of side effect is probably resulted from getting inadequate counseling during medical advice from health care providers and women decreased knowledge of anemia and IFAS. Through better counseling about the overall aspect of tablets, non-adherence due to fear of side effects could be decreased. The finding is consistent with studies done in Kenya [20], Pakistan [22] and Ethiopia [28, 29]. Taking the tablets will lead to too big baby and the tablets would harm the mother and the infant were the other reasons of pregnant women for missing the tablets. This could be due to health care provider's inadequate counseling about the tablets and pregnant women decreased knowledge of iron and folic acid supplementation and anemia. This finding is supported by other studies in Ethiopia [6, 12], and Pakistan [22]. Since evidence on adherence status was from mothers' individual report of supplement intake, recall bias is one of the most important limitations of this study. 


\section{CONCLUSION}

The rate of adherence was found to be low. Maternal educational status, early registration for ANC, history of anemia during current pregnancy and knowledge of mothers about IFAS were predictors of adherence to IFAS. Getting medical advice followed by fear of illness if missed were the primary reasons that enforce mothers to take the tablets. On the other hand, forgetfulness and fear of side effects were among the prominent reasons for missing the doses of tablets. Hence, improving mothers' knowledge regarding overall aspects of the tablet through better advice, community teaching and the mass media at large, would improve adherence.

\section{ACKNOWLEDGEMENTS}

Our heartfelt thank goes to Haramaya University, College of Health and Medical Sciences, School of Graduate Studies for permission and support to conduct the study. We are also thankful to the study subjects for their participation.

\section{REFERENCES}

[1] De Benoist B., E. McLean, I. Egli, Wojdyla D, M. Cogswell, "Worldwide prevalence of Anemia 1993-2005, PubMed | Google Scholar, 2008. [Online], Aviable : Geneva:http://apps.who.int/iris/bitstream/10665/43894/1/9789241596657

[2] Yekta, Z., R. Pourali, N. Mladkova, M. ghasemi-rad, F. Boromand and K. Tappeh, "Role of iron supplementation in promoting maternal and fetal outcome," Therapeutics and Clinical Risk Management, vol. 7, pp. 421-42, 2011.

[3] CSA (Central Statistical Agency). Addis Ababa, Ethiopia and Calverton, Maryland, USA:, Ethiopia Demographic and Health Survey. Central Statistical Agency and ICF International, 2011, [Online], Aviable: http://dhsprogram.com/pubs/pdf/FR255/FR255.pdf: PubMed | Google Scholar.

[4] WHO (World Health Organization), "Haemoglobin concentrations for the diagnosis of Anemia and assessment of severity," Geneva, Switzerland, WHO, 2011.

[5] Mithra, P., B. Unnikrishnan, T. Rekha, K. Nithin, K. Mohan, V. Kulkarni, et al., "Compliance with iron-folic acid (IFA) therapy among pregnant women in an urban area of south India," African Health Sciences, vol. 14, no. 1, pp. 255-260, 2014.

[6] Bekele Taye, Gedefaw Abeje and Alemetsehaye Mekonen, "Factors associated with compliance of prenatal iron folate supplementation amongwomen in Mecha district, Western Amhara: a cross-sectional study," Pan African Medical Journal, vol. 20, no. 43, 2015.

[7] WHO (World Health Organization). Guideline: Daily iron and folic acid supplementation in pregnant women. Geneva, Switzerland, WHO, 2012.

[8] FMOH (Fedreal Ministry of Health), "Implementation Guidelines for the Prevention and Control of Micronutrient Deficiencies," FMOH, Ethiopia, 2014.

[9] Haidar, J. A. and R. S. Pobocik, "Iron deficiency anemia is not a rare problem among women of reproductive ages in Ethiopia: a community based cross sectional study," BMC Blood Disorders, PubMed | Google Scholar, 2009. doi:10.1186/1471-2326-9-7

[10] Burke, R., J. Leon and P. Suchdev Identification, "Prevention and Treatment of Iron Deficiency during the First 1000 Days," Nutrients, vol. 6, pp. 4093-4114, 2014.

[11] Wendt, A., R. Stephenson, M. Young, A. WebbGirard, C. Hogue and U. Ramakrishnan, "Individual and Facility-Level Determinants of Iron and Folic Acid Receipt and Adequate Consumption among Pregnant Women in Rural Bihar," PloS, vol. 10, no. 3, India 2015.

[12] Fiedler, Jack, D’Agostino, Alexis, Sununtnasuk and Celeste, "Nutrition Technical Brief: A Rapid Initial Assessment of the Distribution and Consumption of Iron-Folic Acid Tablets through Antenatal Care in Ethiopia," 2014.

[13] Abel Gebre, Mulugeta Afework and Etana Belachew, "Assessment of Factors Associated with Adherence to Iron-Folic Acid Supplementation Among Urban and Rural Pregnant Women in North Western Zone of Tigray, Ethiopia: Comparative Study,” International Journal of Nutrition and Food Sciences,4(2): 161-168, 2015.

[14] Birhanu TM., Birarra MK., and Mekonnen FA., "Comploiance to Iron and folic acid supplementation in pregnancy, Nortwest Ethiopia,” BMC Res Notes, vol. 11, no. 1, pp. 345, 2018. Doi:10.1186/s13104-018-34-33-3

[15] Tsegaye Molla, Tadesse Guadu, Esmael Ali Muhammad and Melkamu Tamir Hunegnaw, "Factors associated with adherence to iron folate supplementation among pregnant women in West Dembia district, northwest Ethiopia: a cross sectional study," BMC Research Note, vol. 12, no. 6, $2019 . \quad$ [Online], Aviable: https://doi.org/10.1186/s13104-019-4045-2; 2019 
[16] Wulandari, L., P. Craig, A. Klinken and Whelan. Foetal Health Locus of Control and iron supplementation adherence among pregnant women in Bali. Journal of Reproductive and Infant Psychology, 2013.

[17] Gashaw Agegnehu, Azeb Atenafu, Henok Dagne, and Baye Dagnew. Adherence to Iron and Folic Acid Supplement and Its Associated Factors among Antenatal Care Attendant Mothers in Lay Armachiho Health Centers, Northwest, Ethiopia, International Journal of Reproductive Medicine, https://doi.org/10.1155/2019/5863737, 2019

[18] Zemenu YK., Tegibelu A., and Zelalem T., "Compliance with iron folic acid and associated factors among pregant women through pill count in Hawassa city, south Ethiopia: a community based cross sectional study," BMC Reproductive Health, vol. 16, no. 1, pp. 14, 2019.

[19] Gebremichael TG, Welesamuel TG, "Adherence to iron-folicacid supplement and associated factorsamong antenatal care attending pregnant mothers in governmental health institutions of Adwa town, Tigray, Ethiopia: Crosssectional study," PLOS ONE, vol. 15, no. 1, 2020. [Online], Aviable: https://doi.org/10.1371/journal.pone.0227090

[20] Dinga, L., A. Mwangi and G. Abong, "Factors associated with adherence to iron/folate supplementation among pregnant women attending antenatal clinic at thika district hospital in kiambu county, Kenya," PubMed | Google Scholar, 2013.

[21] Mary K., Mirie., and Samuel K., "Compliance with Iron and Folic Acid supplementation (IFAS) and associated factors among pregnant women: results from a cross-sectional study in Kiambu, Kenya," BMC Public Health, vol. 18, no. 1, 2018. Doi:10.1186/s12889-018-5437-2

[22] Nisar, Y., A. Alam, B. Aurangzeb and M. Dibley, "Perceptions of antenatal iron-folic acid supplements in urban and rural Pakistan: a qualitative study," BMC Pregnancy and Childbirth, vol. 14, no. 344, 2014.

[23] Lacerte, P., M. Pradipasen, P. Temcharoen, N. Imamee, Thavatchai and Vorapongsathorn, "Determinants of Adherence to Iron/Folate Supplementation During Pregnancy in Two Provinces in Cambodia," Asia-Pacific Journal of Public Health, vol. 23, no. 3, pp. 315-323, 2011.

[24] Titaley, C. R. and M. J. Dibley, "Antenatal iron/folic acid supplements, but not postnatal care, prevents neonatal deaths in Indonesia: analysis of Indonesia Demographic and Health Surveys 2002/2003-2007, a retrospective cohort study," BMJ, vol. 2, no. 6, 2012.

[25] Abdullahi, H., G. Gasim, A. Saeed, A. Imam and I. Adam, "Antenatal iron and folic acid supplementation use by pregnant women in Khartoum, Sudan," BMCPublic Health, vol. 7, no. 498, 2014.

[26] Ogundipe, O., C. Hoyo, T. stbye, O. Oneko, R. Manongi, R. Lie, et al., "Factors associated with prenatal folic acid and iron supplementation among 21,889 pregnant women in Northern Tanzania: A cross-sectional hospital-based study," BMC Public Health, vol. 12, no. 481, 2012. [Online], Aviable: http://www.biomedcentral.com.

[27] Gebremariam AD., Tiruneh SA., Abate BA., Engidaw MT., Asnakew DT., "Adherence to iron with folic acid and its associated factors among pregant women attending antenatal care follow up at Debre Tabor General Hospital, Ethiopia, 2017," Plose One,vol. 4, no. 1, 2019.

[28] Samson Gebremedhin, Aregash Samuel, Girma Mamo, Tibebu Moges and Tsehai Assefa, "Coverage, compliance and factors associated with utilization of iron supplementation during pregnancy in eight rural districts of Ethiopia: a cross-sectional study," BMC Public Health, vol. 14, 2014.

[29] Abinet Arega , Lakew Abebe, and Mamusha Aman, "Compliance with Iron-Folate Supplement and Associated Factors among Antenatal Care Attendant Mothers in Misha District, South Ethiopia: Community Based Cross Sectional Study," Journal of Environmental and Public Health, 2015. http://dx.doi.org/10.1155/2015/781973 